\title{
ANÁLISE DA GEOMETRIA DE UM TÚNEL DE VENTO PARA CALIBRAÇÃO DE ANEMÔMETROS
}

\author{
André Luiz MelgaÇo Lopes, \\ ÍcAro Freires SousA, Francisco Frederico dos SANTOS MATos \\ Instituto Federal de Educação, Ciência e Tecnologia do Ceará (IFCE), \\ Campus de Maracanaú \\ $<$ andremelgaco14@gmail.com> \\ $<$ icarofreiressousa@gmail.com>, <francisco.f.matos@gmail.com>
}

DOI: 10.21439/conexoes.v10i5.1169

\begin{abstract}
Resumo. Anemômetros são equipamentos utilizados para medir a velocidade do vento. Sua calibração é de grande importância, uma vez que influencia diretamente na estimativa do potencial eólico, sendo assim, decisivo na avaliação de investimentos em empreendimentos eólicos. Portanto, é extremamente importante que esses equipamentos sejam periodicamente calibrados, seguindo parâmetros específicos. Nesse contexto, a norma IEC 61400-12-1 estabelece parâmetros de calibração anemométrica. Para verificar os pré-requisitos estabelecidos na norma citada, este trabalho avaliou o desempenho de uma geometria, normalmente utilizada em túneis de vento, através da simulação da dinâmica dos fluidos no interior do túnel, onde foram propostas alterações com a finalidade de melhorar tais parâmetros. O software Ansys-CFX 12.1 foi utilizado como ferramenta computacional para obtenção dos campos de escoamento em quatro casos específicos, onde foram analisadas duas geometrias e dois tipos de escoamentos na entrada do túnel. Uma análise comparativa entre esses casos aponta quais foram os melhores resultados, e demostra como sutis alterações no formato da geometria podem aproximar em mais de $100 \%$ as condições do túnel aos parâmetros da norma.
\end{abstract}

Palavras-chaves: Túnel de vento. Anemômetro. Calibração. Simulação. Geometria.

\begin{abstract}
Anemometers are equipment used to measure wind speed. Its calibration is of great importance, since it directly influences the estimation of the wind potential, being thus decisive in the evaluation of investments in wind farms. Therefore, it is extremely important that these equipment be periodically calibrated, following specific parameters. In this context, the IEC 61400-12-1 standard establishes anemometric calibration parameters. In order to verify the prerequisites established in the cited standard, this work evaluated the performance of a geometry commonly used in wind tunnels, through the simulation of the fluid dynamics inside the tunnel, where changes were proposed in order to improve such parameters. The Ansys-CFX 12.1 software was used as a computational tool to obtain the flow fields in four specific cases, where two geometries and two types of flow were analyzed at the entrance of the tunnel. A comparative analysis of these cases indicates which were the best results, and shows how subtle alterations in the geometry format can approximate in more than $100 \%$ the tunnel conditions to the standard parameters.
\end{abstract}

Keywords: Wind tunnel. Anemometer. Calibration. Simulation. Geometry.

\section{INTRODUÇÃO}

A campanha de levantamento do potencial eólico é um processo decisivos para qualificar os recursos energéticos e a rentabilidade da implantação de um parque eólico. Para isso, são utilizados anemômetros, aparelhos que medem a velocidade do vento, sendo assim amplamente utilizados nos empreendimentos eólicos.

No mercado atual, existem três principais tipos de anemômetros: os de copos, de hélice e os sônicos. Onde 
se destaca o anemômetro de copos, graças a sua alta confiabilidade e boa relação custo benefício (ALÉ; SIMIONI; HACK, 2008).

A produção de energia eólica é diretamente proporcional ao cubo da velocidade do vento, graças a isso, um erro de medição na velocidade terá a mesma proporção, o que leva a indústria eólica adotar rigorosos padrões de qualidade para tais medições, com a finalidade de garantir a menor margem de erro possível, e consequentemente, um maior percentual de confiabilidade.

A confiabilidade das medições na indústria eólica são fatores decisivos para o processo de financiamento de um parque eólico, onde os bancos internacionais buscam assegurar que a produção energética estimada seja a mais precisa possível. Para tal. São exigidos que durante toda campanha de levantamento do potencial eólico sejam utilizados anemômetros com certificado de calibração (EMDEN, 2016). No Brasil, tais parâmetros são cobrados pela EPE (Empresa de Pesquisa Elétrica), juntamente ao MME (Ministério de Minas e Energia).

Um exemplo que demonstra o quão importante é a calibração anemométrica, são os cálculos de Albers e Klug (1999) para o levantamento do potencial energético, usando um anemômetro com uma incerteza de $\pm 0,5 \mathrm{~m} / \mathrm{s}$, onde a velocidade média é $7 \mathrm{~m} / \mathrm{s}$. A incerteza na produção de energia será em mais de $21 \%$ para mais ou para menos. Segundo o mesmo autor, isso acontece porque as medições nos empreendimentos eólicos dependem constantemente da utilização de anemômetros, gerando assim, erros em cadeia. O que torna a calibração do anemômetro um fator decisivo para avaliar a rentabilidade de um empreendimento.

A norma IEC 61400-12-1 (2005), estabelece que a calibração do anemômetro de copos, deve ser realizada em fluxo uniforme, horizontal e em regime estacionário para níveis de baixa turbulência. Tais condições controladas, são atingidas mais facilmente em um túnel de vento específico para calibração desses instrumentos, que por sua vez, também é regulamentado pela mesma norma citada, onde são estabelecidas as características que um túnel de vento deve atender, elas estão presentes na Tabela 1

Nesse trabalho, foi utilizado geometria de um túnel de vento de seção aberta para executar simulações computacionais e realizar analises quantitativa em CFD (Computational Fluid Dynamics). As dimensões tiveram como referência o trabalho da PUCRS, de ??), no qual foi avaliado um túnel de seção aberta do tipo soprador, específico para calibração de anemômetros, com 12 metros de comprimento e uma seção de testes de 1 metro quadrado, semelhante as geometrias representadas na Figura 1

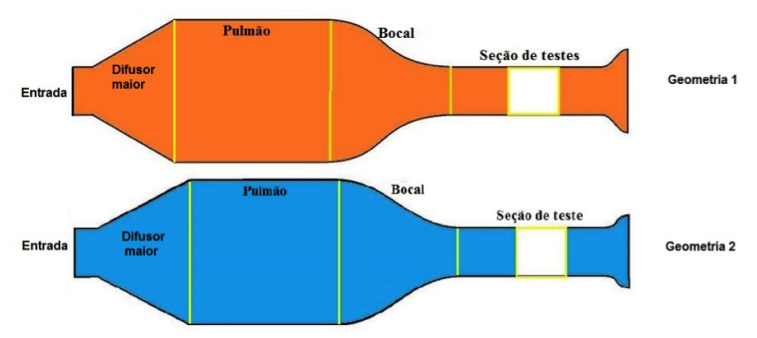

Figura 1: Os túneis, laranja e azul, representam o túnel 1 e 2 respectivamente

Fonte: O autor

O objetivo deste trabalho foi estudar o comportamento do fluxo do ar dentro do túnel de vento, visando otimizar a geometria do mesmo, e em seguida, fazer uma análise comparativa dos resultados coletados através de simulações em CFD com os quesitos da norma.

\section{MATERIAIS E MÉTODOS}

Para atender os objetivos do trabalho, foram criadas duas geometrias (1 e 2, ilustradas na Figura 1) que se diferenciam por dois aspectos. O primeiro, foi a mudança na construção da curva do bocal, onde na geometria 1 foi construída utilizando uma curva de Bezier, e a geometria 2 com um polinômio de quinto grau, mostrado na Eq. (1) (e aplicado na Figura 2). Segundo Vincensi (2014), esse polinômio é resultado de um trabalho realizado em 1988 por Bell e Mehta, que fizeram análises em diferentes curvas de contrações projetadas utilizando polinômios de terceiro, quinto, e sétimo grau. A segunda diferença foi na geometria 2 , que teve um aumento no comprimento do difusor, localizado antes do pulmão, com a finalidade de fazer o fluido se expandir de maneira mais suave.

$Y\left(X^{\prime}\right)=H i-(H i-H e)\left[6\left(X^{\prime}\right)^{5} 15\left(X^{\prime}\right)^{4}+10\left(X^{\prime}\right)^{3}\right]$,

O método numérico empregado para solução das equações da mecânica dos fluidos (FOX; PRITCHARD; MCDONALD, 2001) foi o de volumes finitos, inicialmente desenvolvido por Patankar (1980). Ele consiste em dividir o domínio em vários volumes de controle, onde a variável de interesse se localiza no centróide do volume. A equação resultante é chamada de 
ANÁLISE DA GEOMETRIA DE UM TÚNEL DE VENTO PARA CALIBRAÇÃO DE ANEMÔMETROS

\begin{tabular}{|c|c|c|c|}
\hline Quesito & $\begin{array}{l}\text { Características do tú- } \\
\text { nel de vento }\end{array}$ & Descrição & Requisitos mínimos \\
\hline 1 & Efeito de Bloqueio & $\begin{array}{l}\text { Relação entre a área do anemômetro mais } \\
\text { frontal e a área total da seção de testes }\end{array}$ & $\begin{array}{l}\text { Não deve exceder } 0.1 \text { na } \\
\text { área de seção de,testes aber- } \\
\text { tas }\end{array}$ \\
\hline 2 & $\begin{array}{l}\text { Uniformidade de es- } \\
\text { coamento }\end{array}$ & $\begin{array}{l}\text { Diferença percentual na velocidade } \\
\text { do,vento dentro do volume da seção de } \\
\text { testes }\end{array}$ & $\begin{array}{l}\text { Menor que } 0,2 \% \text { nas dire- } \\
\text { ções, longitudinal, transver- } \\
\text { sal e vertical }\end{array}$ \\
\hline 3 & $\begin{array}{l}\text { Gradiente de Vento } \\
\text { Horizontal }\end{array}$ & $\begin{array}{l}\text { Pressão dinâmica diferencial na } \\
\text { área,coberta pelo anemômetro }\end{array}$ & Deve ser menor que $0,2 \%$ \\
\hline 4 & $\begin{array}{l}\text { Intensidade da Tur- } \\
\text { bulência }\end{array}$ & $\begin{array}{l}\text { Relação entre padrão de velocidades } \\
\text { do,vento e o Desvio padrão da velocidade }\end{array}$ & Deve ser menor que $2 \%$ \\
\hline
\end{tabular}

Fonte: Coquilla (2009).

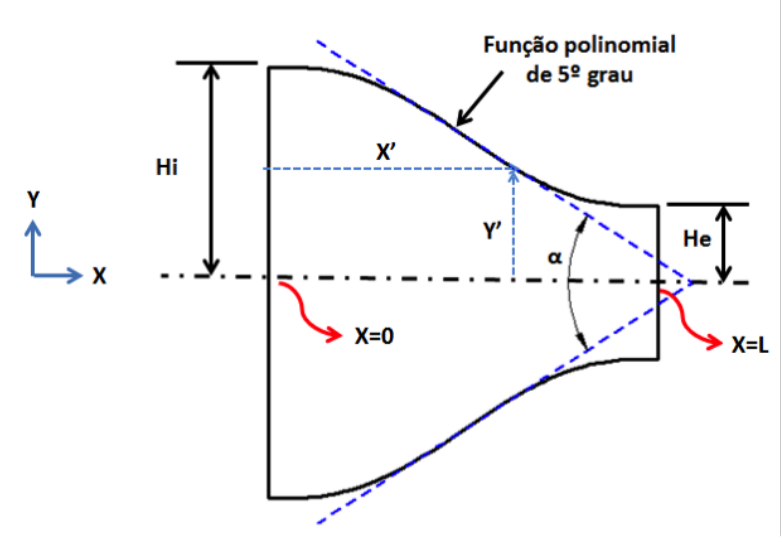

Figura 2: Contração definida por polinômio de $5^{\circ}$ grau Fonte: Vincensi (2014)

equação discretizada, que expressa o princípio da conservação da massa, quantidade de movimento e energia dentro do volume. Para tanto, as geometrias foram criadas no software de CAD Inventor, Auto Desk, e exportadas para o Ansys-Icem, onde foi gerada uma malha com diversos volumes de controle. Na sequência, essa malha foi utilizada no Ansys-CFX para inserção das condições de contorno nas fronteiras do domínio e o campo de escoamento foi obtido com a solução no Ansys-CFX-Solver.

A seguir, foram abordadas as metodologias aplicadas no projeto. A primeira foi a análise qualitativa da malha, para garantir resultados confiáveis e economizar recursos computacionais. Em seguida, as condições para configuração foram definidas, organizadas em casos. Por fim, foi demonstrado como ocorreram os cálculos dos parâmetros para analisar o desempenho da ge- ometria, dada a simulação executada.

\subsection{Análises qualitativa da malha}

A malha criada no Ansys-Icem tende a apresentar resultados mais precisos conforme aumenta-se o refino. Entretanto, o refinamento excessivo gera uma simulação cada vez mais pesada, tornando inviável a esquematização de várias simulações, e por isso, é muito importante encontrar uma malha que atenda às necessidades de precisão necessária e não seja excessivamente refinada. Para isso, foram criadas três malhas, a primeira mais grosseira, com 360.000 nós, outra mais refinada, com 663.255 nós, e por fim, uma malha com o maior refino possível, isto é, no limite dos recursos computacionais em função do processador utilizado, ficando com 2.592.000 nós. As malhas foram chamadas de grosseira, intermediária e extremamente refinada, respectivamente. Por fim, executando uma simulação, foi gerada uma linha vertical dentro da seção de testes, onde foi plotado um gráfico da altura em função da velocidade do vento. Ver Figura 3 .

Note que o resultado da malha intermediária basicamente inscreve o da malha extremamente refinada. Com isso, pode-se adotar esse modelo de malha para qualquer simulação sem perdas significativas na precisão e um tempo computacional razoável.

\subsection{Condições de simulação}

Após a construção da malha, definiu-se as condições de simulação no software Ansys CFX-Pre. De maneira geral, todas as simulações tiveram em comum duas características: primeiro o domínio computacional, onde foi definido como ar à $25^{\circ} \mathrm{C}$ e pressão absoluta de $1 \mathrm{~atm}$; 


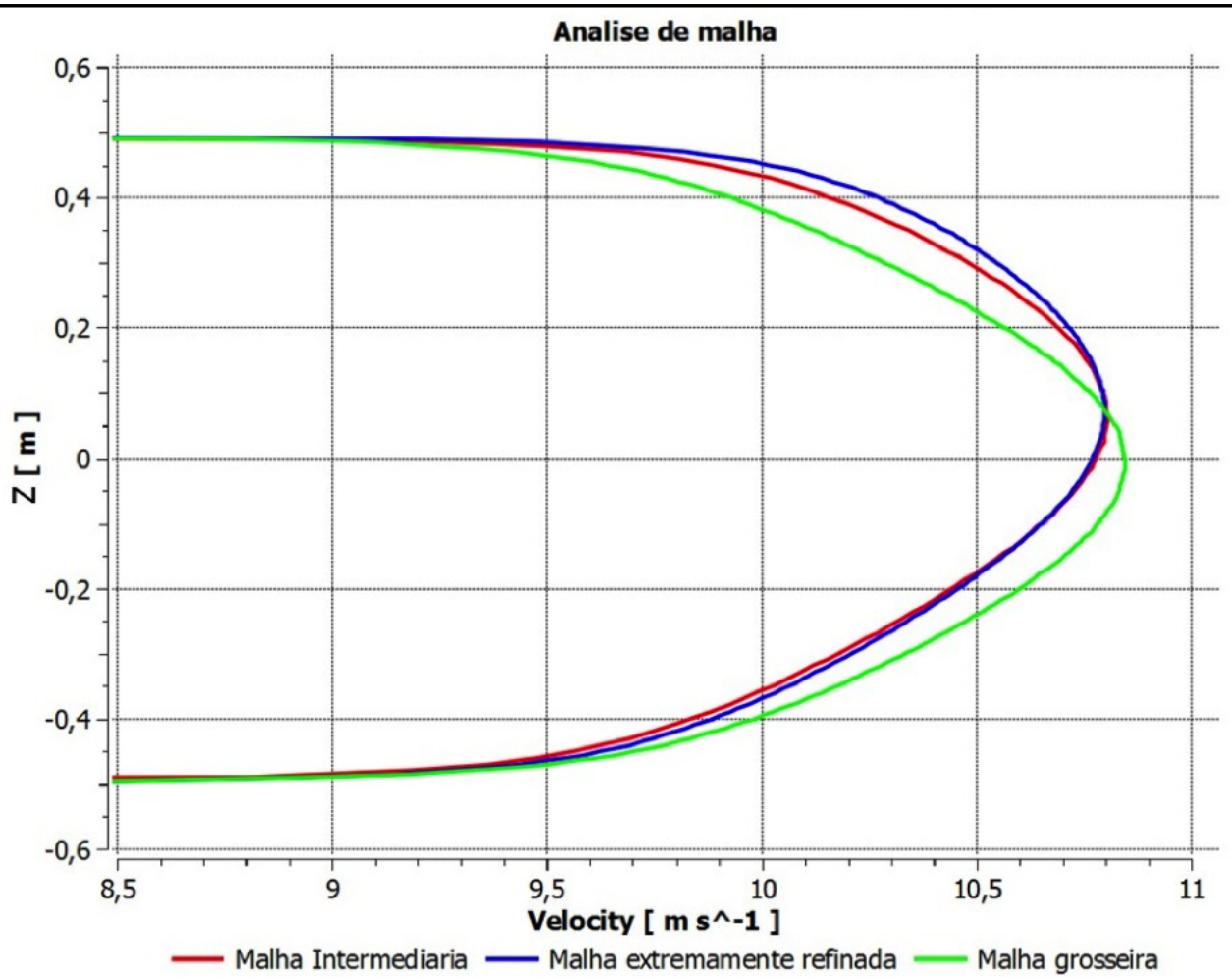

Figura 3: Altura da seção de testes em função da velocidade v

Fonte: $\mathrm{O}$ autor

Segundo, a condição de contorno na saída foi definida com pressão relativa de 0 atm.

O que diferem as simulações realizadas são as condições de contorno na entrada, sendo uma uniforme e a outra não uniforme, onde a segunda se aproxima da não uniformidade de um campo de velocidade gerado por um ventilador do tipo radial. Com base no trabalho de Ledo, M. e Maia (maio, 2014), foi observado que o escoamento na saída de um ventilador soprador de um túnel de vento tende a se concentrar na parede superior do túnel. Portanto, foi idealizada uma condição de contorno na entrada da geometria do túnel (ou saída do ventilador) para levar em consideração a não uniformidade observada. O polinómio de terceiro grau, Eq. (2), foi utilizado para isso, onde a velocidade perpendicular na entrada varia em função do eixo $Z[\mathrm{~m}]$, sendo zero nas paredes do túnel, e tendo seu ponto de máximo a dois terços da altura do bocal de entrada. A constante "a", da Eq. (2), é $120 \mathrm{~Hz}$, garantindo uma velocidade média na seção de teste de $10 \mathrm{~m} / \mathrm{s}$.

$$
V(z)=a\left(z^{2}-z^{3}\right)
$$

A Figura 4 ilustra como varia o campo de velocidade, inscrito pela Eq. (2), que define a condição de contorno na entrada do túnel.

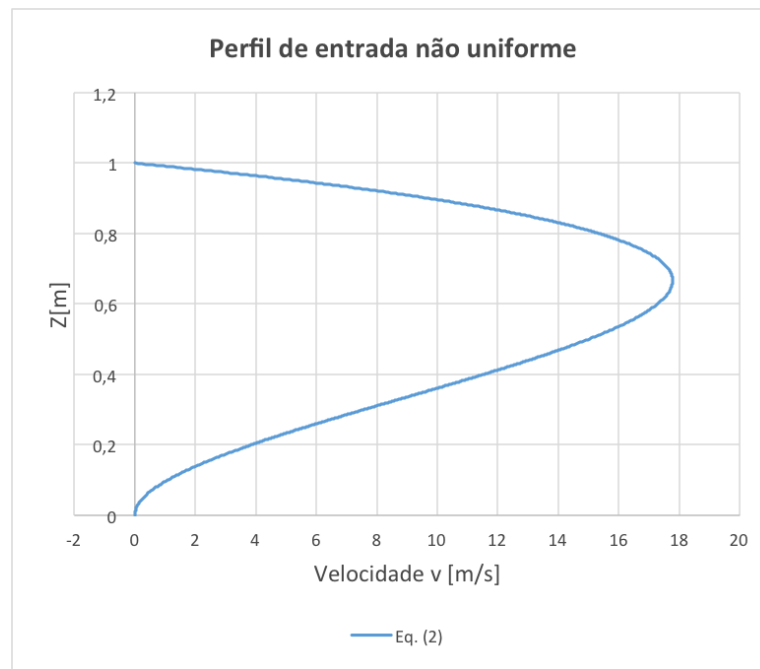

Figura 4: Campo de velocidade da entrada do túnel de vento, conforme Eq. 2)

Fonte: O autor

Dadas as duas geometrias e as condições de entrada, foram distribuídos, de forma esquemática, 4 casos de 
simulações, conforme a Tabela 2

Tabela 2: Esquemas de simulações

\begin{tabular}{lcl}
\hline \multicolumn{3}{c}{ Distribuição das simulações } \\
\hline Casos & Geometria & $\begin{array}{l}\text { Condição de con- } \\
\text { torno na entrada }\end{array}$ \\
\hline CASO A & 1 & $\begin{array}{l}\text { Uniforme } \\
\text { Não uniforme }\end{array}$ \\
CASO B & 2 & $\begin{array}{l}\text { Uniforme } \\
\text { Não uniforme }\end{array}$ \\
\hline CASO C & 2 & CASO D \\
\hline
\end{tabular}

Fonte: O autor

\subsection{Cálculos das características do túnel}

Para calcular os parâmetros da Tabela 1, foi gerado um volume virtual esférico de $30 \mathrm{~cm}$ de diâmetro (tamanho apropriado para cobrir perfeitamente a área de um anemômetro), localizado no centro da seção de testes, conforme a Figura 5, e todos os cálculos posteriores foram realizados pelo dentro do volume criado.

$\mathrm{O}$ efeito de bloqueio demostrado pela Eq. (3), é a razão entre a área mais frontal do anemômetro (An) e a área da seção de testes $(\mathrm{St})$. Esse parâmetro é exigido pela norma IEC 61400-12-1, e garante que a seção de testes tenha uma área suficientemente grande em relação ao tamanho do anemômetro.

$$
\frac{A n}{S t}<0,1,
$$

O quesito 2 da Tabela 1 se refere da uniformidade de escoamento, sendo esse o coeficiente de variância da velocidade no sentido u, v e w. Conforme mostrado na Eq. (4), onde "Vp" é a velocidade pontual, isto é, a velocidade em um nó da malha, "Vm" é a velocidade média dentro do volume, e "n", é o número total de nós dentro da esfera ilustrada na Figura 5 .

$$
\frac{\sqrt{\frac{\sum_{I=1}^{n}(V p-V m)^{2}}{n-1}}}{V m} \cdot 100<0,2 \%,
$$

O gradiente de escoamento, conforme descrito na no quesito 3 da Tabela 1. é representado pela Eq. (5), onde "Dp" é a pressão dinâmica pontual e "Dm", é a pressão dinâmica média.

$$
\frac{\sqrt{\frac{\sum_{i=1}^{n}(D p-D m)^{2}}{n-1}}}{D m} \cdot 100<0,02 \%,
$$

A intensidade de turbulência é representada pela Eq. (6), onde "T" é a média de energia cinética turbulenta. Ela representa o quesito 4 da Tabela 1

$$
\frac{T}{\frac{1}{2} V m^{2}} \cdot 100<2 \%,
$$

Dessa forma, foram aplicadas as Eqs. (3r??) para calcular todos os parâmetros exigidos por norma, mencionados na Tabela 1

\section{RESULTADOS E DISCUSSÕES}

Dadas todas as condições de simulação satisfeitas, foi feita uma análise estatística visual dos resultados, onde primeiramente, foi criado um plano YZ que corta a geometria no centro, e em seguida é apresentada uma vista isométrica, onde são visualizadas as linhas de escoamento do fluido, conforme as Figura $6 \mathrm{e} 7$ Nos dois casos, as cores representam a intensidade da velocidade do vento.

A Figura 6 se refere a simulação do caso $C$, onde pode ser observado, através do plano central, que o vento tende a perder velocidade no pulmão do túnel. Isso acontece devido ao aumento da área de escoamento do fluxo, e tem como objetivo reduzir a turbulência gerada pelo ventilador.Ainda na mesma figura, pode-se observar as linhas de corrente, que demostram uma recirculação de ar dentro do pulmão da geometria.

A Figura 7 se refere a simulação do caso $\mathrm{D}$, onde se pode observar, no plano central, uma não uniformidade da velocidade do vento na entrada da geometria, e após passar pelo pulmão, foi obtido um fluxo mais uniforme. Isso acontece porque o pulmão permite um melhor acomodamento do fluxo de ar, e em seguida, o bocal comprime suavemente o mesmo fluxo, o tornado mais uniforme. Nas linhas de correntes da Figura 7 obteve-se uma recirculação dentro do pulmão bem mais intensa que o da Figura 6

A Tabela 3 apresenta os resultados obtidos para os casos A, B, C e D, definidos na Tabela 2, aplicando os métodos de cálculo das características do túnel, através das Eqs. (3) 6).

Dados os resultados apresentados, pode-se notar que o efeito de bloqueio foi constante, isso acontece porque não se variou a área frontal do anemômetro e nem a da seção de teste. No mais, nota-se que os casos que mais se aproximam das exigências da norma foram o A e C, pois se tratam de casos ideais, onde o fluxo já iniciava de maneira uniforme, gerando assim resultados mais regulares. Porém, os resultados do CASO C conseguiram ser até 4 vezes melhor do que o CASO A (nos quesitos 2 e 3 da Tabela 1), demonstrando que a geometria 2 consegue ser mais eficiente que a 1 , graças a curva do bocal descrita pela a Eq.(1), pois descreve 


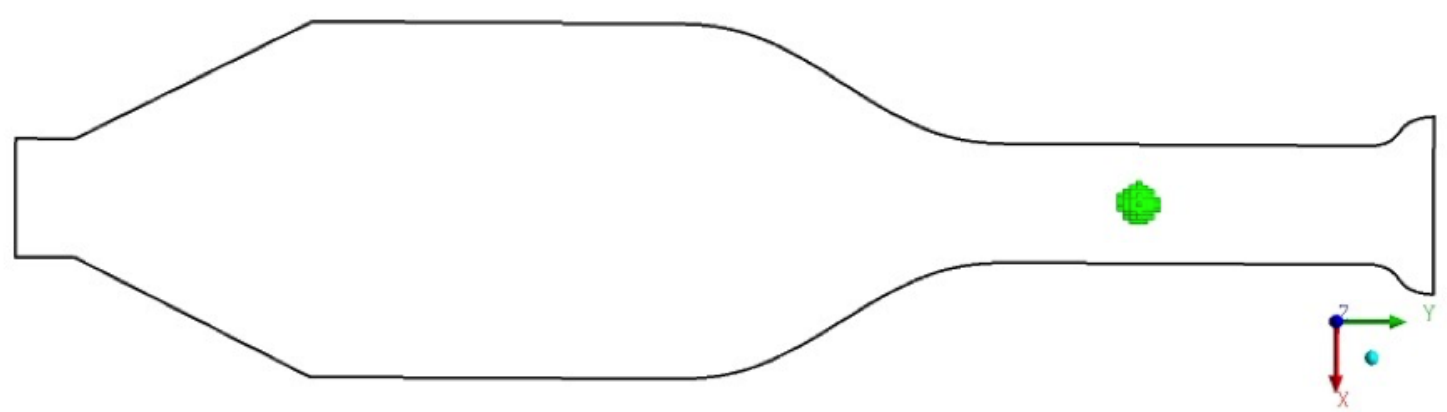

Figura 5: Perfil da geometria do túnel de vento com uma esfera de $30 \mathrm{~cm}$ de diâmetro criada na seção de testes Fonte: O autor

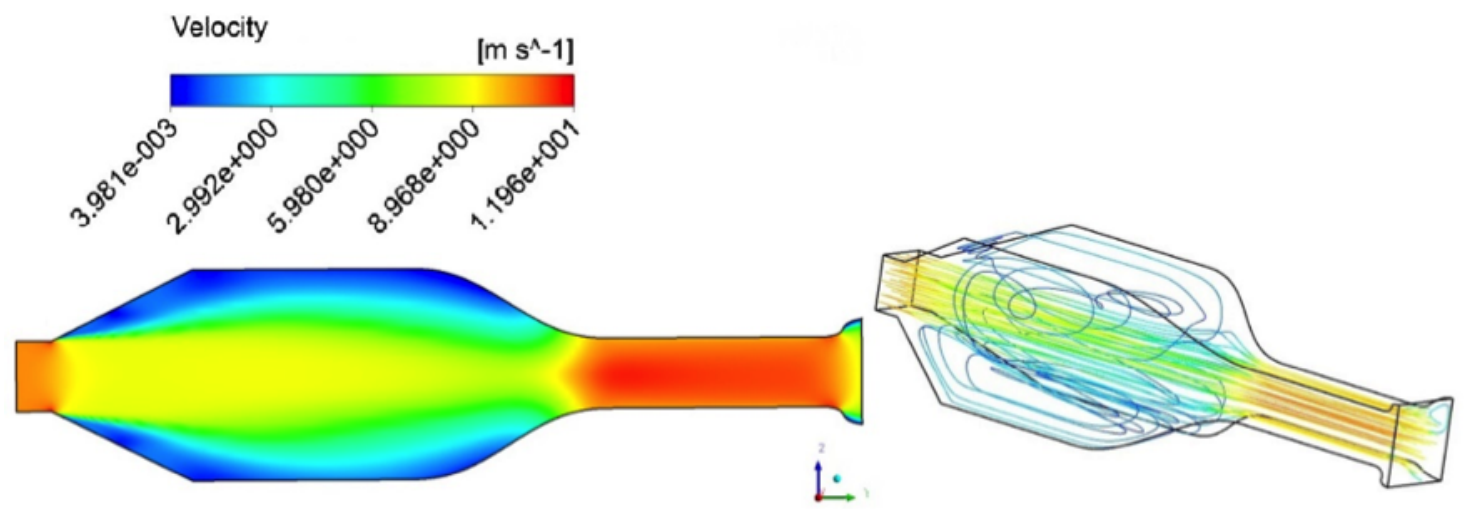

Figura 6: Velocidade do vento em um plano central e as linhas de corrente dentro do túnel de vento: Simulação do Caso C Fonte: $\mathrm{O}$ autor

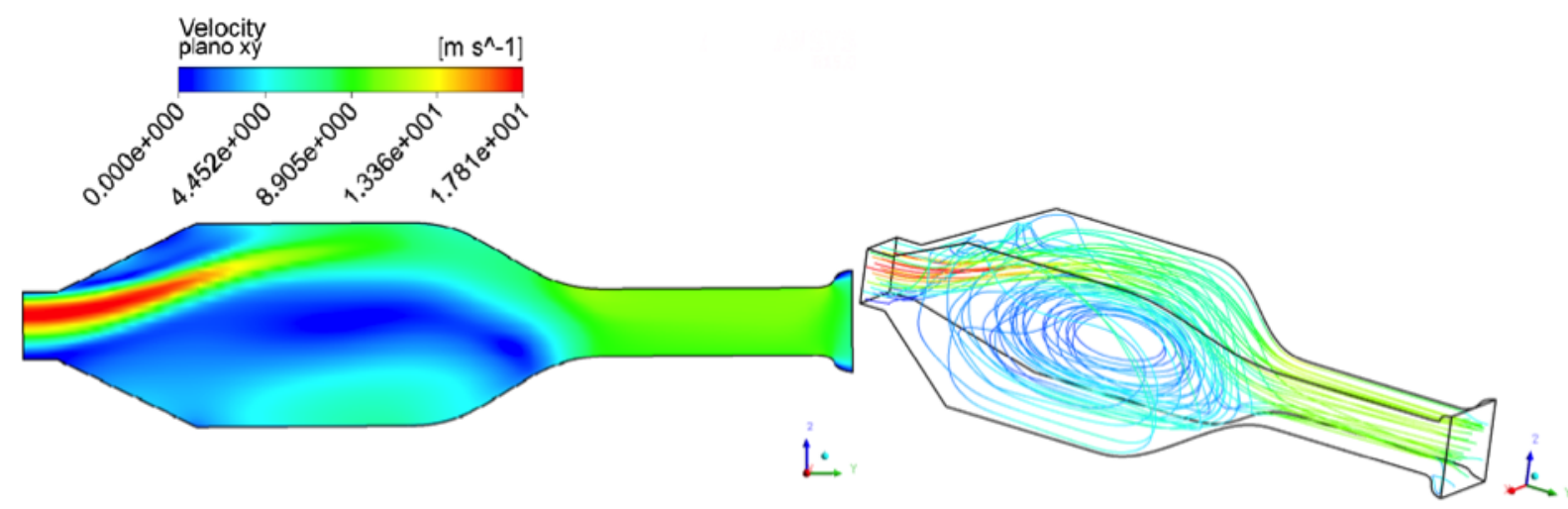

Figura 7: Velocidade do vento em um plano e o refluxo dentro do pulmão: Simulação do caso D 


\begin{tabular}{|c|c|c|c|c|c|}
\hline \multicolumn{6}{|c|}{ Análise de resultados } \\
\hline \multirow{2}{*}{\multicolumn{2}{|c|}{$\begin{array}{l}\text { Quesito / } \\
\text { Esperado }\end{array}$}} & \multicolumn{4}{|c|}{ Resultado } \\
\hline & & CASO A & CASO B & CASO C & CASO D \\
\hline Efeito de bloqueio $/<0,1$ & & $0,0636 \%$ & $0,064 \%$ & $0,063 \%$ & $0,064 \%$ \\
\hline \multirow{4}{*}{ Uniformidade de escoamento $/<0,2$} & Velocidade u & $1,953 \%$ & $0,941 \%$ & $0,481 \%$ & $0,920 \%$ \\
\hline & Velocidade $\mathrm{v}$ & $0,183 \%$ & $0,556 \%$ & $0,109 \%$ & $0,575 \%$ \\
\hline & Velocidade w & $0,207 \%$ & $0,827 \%$ & $0,637 \%$ & $0,800 \%$ \\
\hline & Velocidade Abs. & $1,950 \%$ & $0,910 \%$ & $0,959 \%$ & $1,695 \%$ \\
\hline \multicolumn{2}{|l|}{ Gradiente de vento horizontal $/<0,2$} & $4,225 \%$ & $1,971 \%$ & $0,959 \%$ & $1,695 \%$ \\
\hline \multicolumn{2}{|l|}{ Intensidade de turbulência $/<2$} & $0,889 \%$ & $1,992 \%$ & $1,121 \%$ & $2,240 \%$ \\
\hline
\end{tabular}

Fonte: $\mathrm{O}$ autor.

uma função mais suave, e mais eficaz na compressão do fluido que passa pelo pulmão. No entanto, o CASO $\mathrm{C}$ perde no quesito 4 da Tabela 1 pois obteve uma intensidade de turbulência aproximadamente $25 \%$ maior em comparação ao CASO A.

Ao comparar os casos onde a condição de entrada do vento foi não uniforme, observou-se que o CASO B obteve resultados próximos ao $\mathrm{D}$ quanto a uniformidade de escoamento. No entanto, o CASO B, conseguiu um gradiente de escoamento horizontal aproximadamente $15 \%$ maior que o CASO D, enquanto o CASO D teve uma intensidade de turbulência quase duas vezes maior que o CASO C.

\section{CONCLUSÕES}

Inicialmente, deve-se notar que poucos dos parâmetros avaliados atingiram os quesitos da norma IEC 61400-12-1, pois os mesmos foram feitos para fins comparativos entre geometria e perfil inicial do vento. Porém, o objetivo do trabalho foi alcançado quando foi demoNstrado que uma pequena alteração na geometria, no caso, a curva do bocal, pode otimizar os parâmetros avaliados.

Contudo, o túnel não apresentou resultados muito distantes aos parâmetros da norma, tendo em vista que o mesmo ainda não está completo devido à ausência de alguns componentes, como a colmeia, que serviria para organizar o sentido do fluxo do vento, e uma tela de estabilização, para amenizar a turbulência.

No trabalho, observar-se que a geometria 2 apresentou resultados melhores quando a condição de entrada é uniforme, como se observa na Tabela 3 , demonstrando que o perfil de escoamento do ventilador representa um fator decisivo na escolha da geometria.
Outro ponto interessante, foi a definição do perfil de escoamento do vento não uniforme, onde o fluxo tende a se uniformizar ao passar pelo bocal, isso mostrar a eficiência do pulmão em "frear" e expandir o fluxo de ar, e a suavidade adequada do bocal em comprimir o mesmo. Contudo, o pulmão gera uma essa recirculação do vento, provocando uma não uniformidade no sentido da velocidade dentro da seção de testes, o que torna necessário a instalação de componentes adicionais no túnel.

\section{REFERÊNCIAS}

ALBERS, A.; KLUG, H. High quality wind speed measurements for site assessment. DEWI Magazine, n. 15 , p. 6-16, 1999.

ALÉ, J. A. V.; SIMIONI, G. C. S.; HACK, P. S. Aspectos da calibração de anemômetros nos empreendimentos eólicos. 1o. Congresso Latinoamericano de Energia do Vento, Montevideo, nov. 2008.

COQUILLA, R. V. Review of anemometer calibration standards. CANWEA, 2009.

EMDEN, V. P. H. Acuracy of wind speed data: a key-factor in the economic analysis of wind energy projects. 2016. Disponível em: <http://www.ekopower.nl/know_how_accur.htm>. Acesso em: 8 out. 2016.

FOX, R.; PRITCHARD, P.; MCDONALD. Introdução à Mecânica dos Fluidos. 5. ed. : LTC, 2001.

IEC 61400-12-1. Wind turbines - Part 12-1: Power Performance Measurements of Electricity Producing Wind Turbines. 1. ed. 2005. 
LEDO, L. F. R.; M., H. S.; MAIA, C. B. Estudo numérico do escoamento de um túnel de vento para baixas velocidade. In: SIMMEC/EMMCOP (Ed.). Juiz de Fora, maio, 2014.

PATANKAR, S. V. Numerical Heat Transfer and Fluid Flow. New York: Hemisphere Publishing Corporation, 1980.

VINCENSI, A. Dimensionamento de um Túnel de Vento Subsônico. Monografia do Curso de Engenharia Mecânica: Universidade Regional do Noroeste do Estado do Rio Grande do Sul, Panambi, 2014. 